\title{
Revisión crítica de la estimulación subtalámica en la enfermedad de Parkinson
}

\author{
J. Guridi; M.C. Rodríguez-Oroz; P. Clavero y M. Manrique
}

Departamento de Neurología y Neurocirugía. Clínica Universitaria. Universidad de Navarra. Pamplona. Navarra.

\section{Resumen}

Los autores realizan una revisión crítica de la estimulación del núcleo subtalámico (NST) en la enfermedad de Parkinson (EP) a largo plazo (3-5 años). La estimulación del NST produce una mejoría significativa de la parte motora de la escala UPDRS (Unified Parkinson Disease Rating Scale) sin medicación a los 3-5 años de la cirugía. Los resultados muestran que los beneficios obtenidos tanto en el temblor, la rigidez, la bradicinesia y las disquinesias, así como la reducción de la medicación están mantenidos significativamente a largo plazo. Otros signos de la enfermedad como la marcha y la estabilidad postural no se mantienen al comparar los beneficios al año de la cirugía. Un porcentaje alto de pacientes intervenidos muestra un deterioro cognitivo durante el seguimiento que puede estar relacionado con la propia evolución de la enfermedad. La conclusión es que la estimulación bilateral del NST continúa siendo efectiva a largo plazo pero debería considerarse la cirugía en un momento más precoz de la evolución de la enfermedad.

PALABRAS CLAVE: Núcleo subtalámico. Enfermedad de Parkinson. Estimulación cerebral profunda. Estimulación subtalámica.

Critical review of the subthalamic stimulation in Parkinson's disease

\section{Summary}

The authors critically review subthalamic nucleus (STN) stimulation for Parkinson's disease (PD) at long follow-up (3-5 years). Subthalamic stimulation induce a significant improvement during the "off" medication in the assessment motor score UPDRS (Unified Parkinson Disease Rating Scale) 3-5 years after surgery. Results

Recibido: 14-05-09. Aceptado: 6-08-09 show that the benefits obtained in tremor, rigidity, bradykinesia, dyskinesias induced by medication and levodopa reduction are significantly maintained during long term. The improvement in other clinical signs as gait and postural stability at long follow-up are not maintained comparing with the benefits obtained one year after surgery. A high percentage of patients show a cognitive disturbance during the follow-up period that may be correlated with the disease progression. The conclusion is that bilateral STN stimulation is an effective treatment for PD patients at long term but it should be considered earlier in the course of PD.

KEY WORDS: Subthalamic nucleus. Parkinson's disease. Deep brain stimulation. Pallidal stimulation. Subthalamic stimulation.

Introducción

La enfermedad de Parkinson (EP) es una enfermedad neurodegenerativa que se caracteriza clínicamente por la aparición de temblor, rigidez y lentitud motora (acinesia o bradicinesia) y que fisiopatológicamente es debido a la progresiva pérdida de neuronas dopaminérgicas (DA) de la sustancia negra compacta ( $\mathrm{SNc}$ ). El tratamiento con levodopa fue introducido a finales de los años 60 del siglo pasado, con inicialmente excelente resultado y esto hizo que la cirugía que entonces se realizaba, talamotomía principalmente, dejara de practicarse. Sin embargo y después de varios años de tratamiento médico, los pacientes presentan complicaciones relacionadas tanto con la medicación como con la progresión de la propia enfermedad, como son las fluctuaciones motoras y disquinesias que incapacitan a los mismos. Esto ha llevado a un renovado interés por los

DBS: deep brain stimulation. EP: enfermedad de Parkinson. GPi: globo pálido interno. MPTP: 1-methyl,4-fenil-1,2,3,6 tetrahidropiridina. NST: núcleo subtalámico. PD: Parkinson's disease. PPN: núcleo pendúnculo pontino. PSP: parálisis supranuclear progresiva. STN: subthalamic nucleus. UPDRS: Unified Parkinson Disease Rating Scale. 
nuevos procedimientos quirúrgicos en los pacientes que presentan este tipo de complicaciones en lo que se define como EP en estado avanzado.

Desde el punto de vista de la diana quirúrgica, la palidotomía (lesión del globo pálido interno) sustituyó a la talamotomía tras mostrar mejores resultados que ésta en la condición parkinsoniana, así como en las disquinesias contralaterales a la lesión. Pero fue posteriormente la aparición de la estimulación cerebral a alta frecuencia de estas dianas lo que verdaderamente permitió el poder realizar cirugías bilaterales en este tipo de pacientes, sin las complicaciones de tipo cognitivo y de lenguaje que ocurrían tras lesiones realizadas en ambos hemisferios. La estimulación cerebral profunda (DBS deep brain stimulation) hasta entonces había sido practicada en pacientes exclusivamente con temblor ${ }^{8,9}$.

El hallazgo en primates a los que se indujo parkinsonismo tras ser tratados con MPTP (1-methyl,4-fenil-1,2,3,6 tetrahidropiridina) mostró que el NST (núcleo subtalámico) mostraba una hiperactividad tanto metabólica como electrofisiológicamente y que la lesión de esta estructura mejoraba la sintomatología motora ${ }^{5,12,13,23,57}$. Esto supuso un hallazgo capital en el entendimiento de la fisiopatología del estado parkinsoniano así como del funcionamiento de los ganglios basales. Benazzouz y cols realizaron una estimulación subtalámica en 2 primates previamente tratados con MPTP mejorando su condición clínica ${ }^{11}$ y en 1993, paralelamente, el grupo de Grenoble publicó los primeros resultados de la estimulación del NST en pacientes con EP con buena respuesta clínica y sin complicaciones ${ }^{10,47}$.

Durante varios años tanto el NST como el GPi (globo pálido interno) han sido las dos dianas quirúrgicas elegidas para mejorar la situación motora de los pacientes con EP avanzada, desplazando a la cirugía talámica que ha quedado reservada para tratamiento del temblor esencial así como de otros tipos de temblores. Sin embargo, en la actualidad, es la estimulación del NST la única cirugía que se mantiene para tratar la EP, mientras que la del GPi se prefiere para el tratamiento de la distonía, corea y de otras disquinesias.

Diferentes centros quirúrgicos en todo el mundo están realizando cirugía en el NST en pacientes con EP con complicaciones motoras y actualmente se están publicando resultados de la estimulación en series de pacientes con un seguimiento postquirúrgico de 4 o 5 años tras la cirugía. En esta revisión se pretende hacer un análisis crítico de diferentes aspectos de la estimulación del NST. En primer lugar, queremos comparar los resultados clínicos entre la estimulación del NST con la del GPi. En segundo lugar, trataremos de actualizar los resultados motores y no motores a largo plazo de los pacientes con estimulación del NST. Por último, se valora una nueva alternativa hacia una cirugía más precoz para este tipo de pacientes.

\section{NST-DBS frente a GPi-DBS.}

La estimulación cerebral profunda tanto en el NST como en el GPi mejora los signos capitales motores de la condición parkinsoniana y reduce las complicaciones inducidas por la medicación ${ }^{7,14,42,49,65}$. Ambas estructuras anatómicas de los ganglios basales, NST y GPi, están hiperactivas en la EP y su inhibición o bloqueo con la estimulación a alta frecuencia induce un claro beneficio motor. Sin embargo, el NST es la diana quirúrgica actualmente preferida por los grupos que realizan estas intervenciones y tres podrían ser las razones para esta elección ${ }^{24,60}$. En primer lugar, los resultados clínicos en los pacientes pueden indicar que el beneficio con NST-DBS es mayor que el obtenido con GPi-DBS, aunque esto no está totalmente constatado por carecer de estudios aleatorizados que comparen ambas dianas quirúrgicas ${ }^{44}$. En segundo lugar y desde el punto de vista neuroquirúrgico, es más fácil definir fisiológicamente durante la intervención quirúrgica con microrregistro y microestimulación, la región sensitivo-motora del NST y sus estructuras adyacentes que la del GPi, que es una estructura anatómica de mayor tamaño y más compleja. Finalmente y no menos importante, la estimulación del NST permite una significativa reducción de levodopa en los pacientes intervenidos, lo que no ocurre en los pacientes implantados en $\mathrm{GPi}$, en los cuales la medicación permanece en las mismas dosis. Este factor es capital sobre todo en los pacientes que presentan problemas motores (disquinesias) y no motores (complicaciones de tipo psiquiátrico) relacionados con la medicación a la hora de elegir una diana sobre la otra ${ }^{24,60}$.

En el artículo se revisan comparativamente los resultados de la estimulación del NST y del GPi que han sido publicados por el mismo grupo de trabajo para una mejor evaluación y comprensión de los resultados clínicos, así como valorar los pros y contras entre ambas dianas quirúrgicas $^{3,34,35,38,39,42,50}$.

\section{Efecto de la cirugía en el "off” medicación}

Los datos clínicos obtenidos en distintos estudios, tanto en el NST como en el GPi, han mostrado una mejoría en los signos capitales de la EP y una reducción significativa en el "off" de la parte motora de la UPDRS (Unified Parkinson's Disease Rating Scale, parte III) unido a una reducción del tiempo "off" con un incremento de las horas "on" sin disquinesias ${ }^{42,44}$. Las intervenciones en el NST han conseguido una reducción significativa de medicación, mientras que en el GPi no conlleva una disminución en la misma. Sin embargo, únicamente hay publicado un estudio aleatorizado que haya comparado las dos dianas quirúrgicas $^{3}$. Por otro lado, los pacientes con GPi-DBS publicados en la literatura, tienen un seguimiento más corto en com- 
Tabla 1

Resultados clínicos de estudios incluyendo estimulación del GPi y NST. Comparación "on" estimulación postoperatoria contra "off” medicación preoperatoria.

\begin{tabular}{|c|c|c|c|c|c|}
\hline Autor & Basal & 6 meses & 12 meses & 3-4 años & 3-4 años vs 1 año \\
\hline \multicolumn{6}{|l|}{$\begin{array}{l}\text { Volkman }^{61} \\
\text { 16NST;11 GPi }\end{array}$} \\
\hline $\begin{array}{l}\text { UPDRS III } \\
\text { STN/GPi }\end{array}$ & $56.4 / 52.5$ & $18.6 / 22.9$ & $22.4 / 16.7$ & & \\
\hline Bradicinesia & $2.5 / 2.5$ & $1.1 / 1.3$ & $1.3 / 1.3$ & & \\
\hline Rigidez & $2.4 / 2.2$ & $0.5 / 1.1$ & $0.6 / 1.1$ & & \\
\hline Temblor & $0.9 / 0.8$ & $0.1 / 0.2$ & $0.1 / 0.2$ & & \\
\hline Ines Postural & $2.7 / 2.2$ & $1.0 / 0.8$ & $1.1 / 0.8$ & & \\
\hline Marcha & $1.7 / 1.3$ & $1.2 / 0.9$ & $1.3 / 0.9$ & & \\
\hline \multicolumn{6}{|l|}{$\begin{array}{l}\text { Krack }^{35} \\
8 \mathrm{STN} ; 5 \text { GPi }\end{array}$} \\
\hline $\begin{array}{l}\text { UPDRS III } \\
\text { NST/GPi }\end{array}$ & $57.3 / 53.6$ & $\begin{array}{l}17.1 / 32.5 \\
p<0.05\end{array}$ & & & \\
\hline Temblor & $4.0 / 4.0$ & $0.5 / 1.6 \mathrm{~ns}$ & & & \\
\hline Rigidez & $13.8 / 13.9$ & $4.5 / 6.8 \mathrm{~ns}$ & & & \\
\hline Acinesia & $19.9 / 19.7$ & $\begin{array}{l}5.7 / 13.7 \\
p<0.05\end{array}$ & & & \\
\hline Marcha & $14.1 / 13.5$ & $\begin{array}{l}3.0 / 8.0 \\
p<0.05\end{array}$ & & & \\
\hline \multicolumn{6}{|l|}{$\begin{array}{l}\text { Anderson }^{3} \\
\text { 10NST:10 GPi }\end{array}$} \\
\hline $\begin{array}{l}\text { UPDRS III } \\
\text { NST/GPi }\end{array}$ & $51 / 50$ & & $\begin{array}{l}27.0 / 30.0 \\
p<0.40\end{array}$ & & \\
\hline Rigidez & $10.5 / 9.5$ & & $\begin{array}{l}5.0 / 5.0 \\
p<0.18\end{array}$ & & \\
\hline Temblor & $9.0 / 7.0$ & & $\begin{array}{l}1.0 / 1.5 \\
p<0.51\end{array}$ & & \\
\hline Bradicinesia & $18 / 15$ & & $10 / 10 p<0.6$ & & \\
\hline Scores axiales & $8 / 7.5$ & & $\begin{array}{l}4.5 / 4.5 \\
p<0.12\end{array}$ & & \\
\hline \multicolumn{6}{|l|}{$\begin{array}{l}\text { Rodriguez-Oroz }^{50} \\
\text { 49NST;20 GPi }\end{array}$} \\
\hline UPDRS III & $56.7 / 51.7$ & & $24.6 / 29.2$ & $28.6 / 31.7$ & $\mathrm{P}<0.02 / \mathrm{ns}$ \\
\hline Temblor & $13.1 / 11.3$ & & $2.3 / 2.7$ & $1.7 / 1.7$ & $\mathrm{Ns} / \mathrm{ns}$ \\
\hline Rigidez & $10.8 / 10.9$ & & $3.8 / 6.5$ & $4.4 / 6.5$ & $\mathrm{Ns} / \mathrm{ns}$ \\
\hline Bradicinesia & $19.9 / 18.3$ & & $10.7 / 10.9$ & $11.3 / 12.9$ & $\mathrm{Ns} / \mathrm{ns}$ \\
\hline Ines Postural & $2.6 / 2.3$ & & $1.2 / 1.4$ & $1.8 / 1.7$ & $\mathrm{P}<0.001 / \mathrm{ns}$ \\
\hline Marcha & $2.8 / 2.5$ & & $1.4 / 1.5$ & $1.7 / 1.8$ & $\mathrm{P}<0.02 / \mathrm{ns}$ \\
\hline
\end{tabular}

paración con los pacientes tratados con NST-DBS en los cuales se han publicado varios estudios con un seguimiento de 4-5 años que consideramos como largo plazo ${ }^{35,38,39,50}$.

Anderson y cols han publicado el único estudio prospectivo y aleatorizado a los 12 meses de la cirugía en 20 pacientes intervenidos (10 con cada diana quirúrgica) ${ }^{3}$
(Tabla 1). Los autores demuestran que tras la cirugía, la puntuación en la UPDRS motora en situación "on" estimulación mejoró en un 39\% en los pacientes intervenidos en el GPi y un $48 \%$ en los pacientes cuya diana era el NST, respecto a la situación "off" preoperatoria, pero la mejoría entre ambos núcleos no fue significativa $(\mathrm{p}<0.40)$. 
El beneficio en el temblor, rigidez y bradicinesia, así como el beneficio axial tampoco mostró significación entre los dos grupos quirúrgicos $(\mathrm{p}<0.12)$. La reducción de las disquinesias ocurrió también en ambas dianas quirúrgicas (89\% para el GPi y $68 \%$ para el NST) mientras que la reducción de levodopa sólo ocurrió en el grupo de pacientes intervenido en el NST. Por tanto, este estudio prospectivo y aleatorizado no ha mostrado diferencias estadísticas entre ambas dianas quirúrgicas ${ }^{3}$ (Tabla 1).

Volkmann y cols retrospectivamente compararon pacientes tratados con NST-DBS (n:16) con pacientes intervenidos con GPi-DBS (n:11) a los 12 meses de la cirugía $^{61}$. La UPDRS motora se redujo significativamente en ambas dianas $(\mathrm{p}<0.005)$, aunque los signos clínicos de las extremidades como el temblor, la rigidez y la bradicinesia sólo mejoraron estadísticamente en los pacientes con estimulación del NST. La postura y la marcha también mejoraron con ambas dianas quirúrgicas, aunque únicamente en el grupo de pacientes intervenidos en el NST la reducción fue significativa. Las disquinesias se redujeron en ambos grupos (GPi $\mathrm{p}<0.005$ y NST $\mathrm{p}<0.001$ ) aunque la disminución de la medicación sólo ocurrió en el grupo de pacientes tratado en el NST ( $65 \%$ de reducción) ${ }^{61}$ (Tabla 1). Un estudio similar respecto a los resultados ha sido publicado por Krause y cols, en un análisis prospectivo y no aleatorizado realizado en 6 pacientes intervenidos en el GPi y en 12 en el NST. Los autores describen un beneficio significativo clínicamente para el grupo de pacientes intervenido en el NST en la mayoría de los items evaluados con las distintas escalas, mientras que la mejora en el grupo de pacientes intervenidos en el grupo de GPi no fue significativa $^{38}$.

Krack y cols compararon 8 pacientes intervenidos con estimulación del NST bilateral con otros 5 pacientes intervenidos en el GPi a los 6 meses de la cirugía, obteniendo una mejoría de la UPDRS motora en ambas dianas quirúrgicas, aunque únicamente el beneficio obtenido en el grupo de pacientes intervenido en el NST fue significativo $(p<0.05)^{35}$. El temblor y la rigidez mostraron similares beneficios en ambas dianas, aunque la bradicinesia mejoró en un mayor porcentaje en el grupo de pacientes intervenido en el NST $(p<0.05)$. Los signos axiales, como la marcha y la estabilidad postural, mejoraron también significativamente con el NST $(p<0.05)$ y no así con el grupo de pacientes intervenido en el GPi ${ }^{35}$ (Tabla 1).

El estudio con un mayor número de pacientes evaluados fue el realizado en 18 centros entre 1995 y 1999 con la participación de 134 pacientes con 96 implantes bilaterales de NST y 38 en el GPi y sus resultados fueron publicados en el $2001^{42}$. Este estudio clínico prospectivo no fue aleatorizado para la diana quirúrgica e incluyó un análisis doble ciego a los tres meses de la cirugía. El mismo, mostró un beneficio con ambas dianas quirúrgicas tras la estimulación y "off" medicación $^{42}$. A los 6 meses, en una evaluación abierta, los pacientes intervenidos en el NST mejoraron las escalas de evaluación en un 74\% $(p<0.001)$ en el tiempo "on" sin disquinesias ( contra un $27 \%$ preoperatoriamente) mientras los pacientes intervenidos con el GPi lo hicieron en un $64 \%$ $(\mathrm{p}<0.01)(28 \% \text { preoperatoriamente })^{42}$. El seguimiento de los pacientes del estudio multicéntrico ha continuado $\mathrm{y}$ ha sido publicado a los 3-4 años postoperatoriamente por Rodríguez-Oroz y cols con 49 pacientes intervenidos en el NST y 20 en el GPi ${ }^{50}$. El análisis de ambas dianas quirúrgicas a los 3-4 años ha sido comparado con la evaluación de los pacientes preoperatoriamente y al año de la cirugía (Tabla 1). Aunque el estudio no se ideó inicialmente para comparar ambas dianas quirúrgicas, las características clínicas de ambos grupos eran similares pudiéndose interpretar como la fuente mayor de datos para poder compararlas a largo plazo. Actualmente este grupo de pacientes ha sido evaluado a los 5-6 años desde la intervención y los datos están pendientes de ser publicados.

La UPDRS motora permaneció significativamente mejorada a los 3-4 años post-cirugía al compararla con la basal prequirúrgica en ambas dianas $(\mathrm{p}<0.0001)$, pero el grupo de pacientes intervenido con NST-DBS mostró un empeoramiento al compararlo con el beneficio obtenido al año de la cirugía $(\mathrm{p}<0.02)$, mientras que el grupo de pacientes intervenido en el GPi-DBS mantuvo el beneficio reportado al año al compararlo con el largo plazo ${ }^{50}$ (Tabla 1). En el estudio, los signos capitales de la enfermedad como el temblor, la rigidez y la bradicinesia mantenían a largo plazo el beneficio observado al año, así como con la basal preoperatoria. En los signos axiales, tales como la marcha o la estabilidad postural, no se mantuvieron las diferencias significativas a largo plazo con las del año postoperatoriamente en el NST mientras sí se mantuvieron en los pacientes intervenidos en el GPi como diana ${ }^{50}$. En cuanto a las disquinesias inducidas por la medicación, el beneficio permanecía a los 3-4 años en ambas dianas quirúrgicas contra el estudio preoperatorio $(\mathrm{p}<0.0001)$, pero no con el beneficio significativo descrito al año de la intervención ${ }^{50}$. La conclusión final del estudio fue que los pacientes mantenían la mejoría motora con la estimulación cerebral con ambas dianas quirúrgicas a largo plazo, pero los pacientes con GPi-DBS mantuvieron el beneficio de forma más estable. El empeoramiento del grupo de pacientes intervenidos con NST-DBS en los parámetros axiales, tales como el balance, la marcha o la estabilidad postural, puede sugerir una progresión de la enfermedad ${ }^{50}$ (Tabla 1).

Un dato importante que debemos constatar, es que el estudio referido no evaluó la calidad de vida de los pacientes o bien otras medidas que pudieran indicar una diferencia basal entre ambos grupos quirúrgicos. Los datos clínicos y las discretas diferencias encontradas entre ambos grupos, deben de ser tomadas con precaución hasta que no 
se realice un nuevo estudio prospectivo ${ }^{27}$. Por tanto, no deberíamos de concluir que la estimulación del GPi pudiera ser superior clínicamente a largo plazo al ser comparada con la estimulación del NST hasta no obtener nuevos datos en este sentido ${ }^{50}$. Otros estudios, por el contrario, publicados en esta línea, han mostraron también una pérdida de eficacia de los pacientes intervenidos con GPi-DBS a largo plazo ${ }^{21,29,62}$. Así Volkmann y cols en 11 pacientes tratados con GPi-DBS bilateral a los 5 años describieron que el beneficio inicial obtenido, tanto motor como sobre las fluctuaciones motoras, disminuyó durante los años de seguimiento e incluso, cuatro pacientes del grupo fueron tratados con estimulación en el NST ${ }^{62}$. Otros autores también reportaron un grupo de pacientes con una mejoría motora inicial tras GPi-DBS que a los 2 años de la cirugía, redujo su eficacia en los diferentes parámetros de las escalas de evaluación y mejoraron tras una nueva cirugía con NST-DBS ${ }^{21,29}$.

\section{Efecto de la estimulación en el "on" medicación}

La evaluación del efecto de la estimulación ("on" estimulación "off" medicación) en ambas dianas quirúrgicas al compararla con el "on" medicación preoperatorio no muestra una mejoría significativa tras la cirugía $a^{3,35,50}$. Esto significa que la intervención quirúrgica no produce ningún beneficio clínico por encima del efecto de la levodopa. Sin embargo, el añadir la medicación postoperatoria a la estimulación tras la intervención ("on/on") mejoran significativamente las escalas motoras de la UPDRS al ser comparada con el efecto de la basal "on" medicación preoperatoria pero únicamente al año de la cirugía, perdiendo esta significación en el análisis a largo plazo ${ }^{35,50}$. Esto ocurre tanto para los pacientes con estimulación del NST como para el grupo del GPi-DBS. Es interesante el referir que este efecto sinérgico de ambos tratamientos no se ha obtenido con ninguna otra terapia aplicada a la EP incluyendo los transplantes dopaminérgicos. Así, en el estudio referido por Rodríguez-Oroz y cols al año de la cirugía $^{50}$, el beneficio motor de la UPDRS en situación "on/ on" fue significativo para el NST-DBS y para el GPi-DBS al ser comparados con el "on" farmacológico preoperatorio. Sin embargo, el análisis a largo plazo mostró un empeoramiento significativo (a los 3-4 años; $\mathrm{p}<0.01$ y 0.05 ) para ambas dianas al ser comparadas con los resultados al año de la intervención. Las escalas todavía mostraban un beneficio al ser comparadas con las basales para ambas dianas quirúrgicas, aunque no fueron significativas ${ }^{50}$. La estabilidad postural y la marcha como signos axiales, se deterioraron con la estimulación del NST, pero permanecieron sin variación en los pacientes tratados con GPi-DBS al evaluar los resultados al año de la cirugía con los resultados a largo plazo $^{50}$.
Por otro lado, todos los estudios han mostrado una reducción de las disquinesias con ambas dianas quirúrgicas y aunque la reducción puede ser estadísticamente equivalente, el mecanismo fisiopatológico puede ser diferente. La reducción de levodopa y su contribución en la reducción de disquinesias ocurre únicamente con la cirugía del NST mientras que no se modifica estadísticamente en los pacientes intervenidos en el GPi, ni al año de la cirugía, ni a largo plazo $3,35,38,39,42,50$. Así el mecanismo antidisquinético de la estimulación del GPi podría ser similar al que también ocurre tras la palidotomía, es decir la interrupción del circuito neuronal a nivel pálido-talámico impidiendo la aparición de movimientos involuntarios ${ }^{25}$. Sin embargo, existe la idea general de que la reducción de las disquinesias tras la estimulación subtalámica está únicamente relacionada con la disminución de medicación ${ }^{31,41,58}$. En este sentido, otros autores, han sugerido que esta reducción de las disquinesias podría estar relacionada con la propia estimulación crónica que pudiera tener un efecto antidisquinético similar al obtenido con la estimulación dopaminérgica continua ${ }^{18,25,36,48}$. Este hecho podría explicar el beneficio disquinético obtenido en los pacientes intervenidos con NST-DBS unilateralmente.

En conclusión, la estimulación de ambas dianas quirúrgicas, NST y GPi, mejora los signos capitales de la EP en los pacientes intervenidos a largo plazo. No se obtiene un beneficio motor de la estimulación sobre la condición "on" preoperatoria aunque las disquinesias disminuyen significativamente al ser comparadas con los resultados "on" en situación basal. Esto hace que exista un incremento en las actividades de la vida diaria (UPDRS II) en la situación "on". Aunque algunos estudios han referido un mayor impacto o beneficio de la cirugía en los pacientes intervenidos en el NST al ser comparados con los resultados obtenidos con GPi-DBS, los datos reportados no lo han mostrado en el único estudio aleatorizado publicado hasta la actualidad ${ }^{3}$.

\section{Resultados de la estimulación del NST a largo plazo}

Recogemos los datos referidos por diferentes grupos quirúrgicos en distintas publicaciones sobre los pacientes intervenidos con estimulación del NST y seguidos mediante escalas de evaluación durante un largo plazo (45 años). Se analizan los resultados motores, así como los efectos adversos derivados de la estimulación y el posible deterioro cognitivo de este grupo de pacientes.

\section{Resultados motores}

Efecto de la estimulación del NST en el "off” medicación

Los resultados muestran que la UPDRS motora así 


\section{Tabla 2}

Resultados quirúrgicos a largo plazo con estimulación del NST y principales signos motores ("on" stimulación contra “off” medicación preoperatoriamente)

\begin{tabular}{|l|l|l|l|l|l|}
\hline & \multicolumn{1}{|c|}{$\begin{array}{c}\text { Krack } \\
\mathrm{y} \mathrm{cols}^{37}\end{array}$} & $\begin{array}{c}\text { Rodriguez-Oroz } \\
\mathrm{y} \mathrm{cols}^{50}\end{array}$ & $\begin{array}{c}\text { Schüpbach } \\
\mathrm{y} \mathrm{cols}^{53}\end{array}$ & $\begin{array}{c}\text { Ostertaag } \\
\mathrm{y} \mathrm{cols}^{45}\end{array}$ & $\begin{array}{c}\text { Visser-Vanderwalle } \\
\mathrm{y} \mathrm{cols}^{59}\end{array}$ \\
\hline $\mathrm{N}^{\mathrm{o}}$ pacientes & 49 & 49 & 37 & 22 & 20 \\
\hline Seguimiento del estudio & 5 & $3-4$ & 5 & 4 & 4 \\
\hline Años de enfermedad & 14.6 & 15.4 & 15.2 & & 15 \\
\hline UPDRS II & $42 \%(0.001)$ & $43 \%(0.0001)$ & $40 \%$ & 0.0001 & $59.4 \%(0.004)$ \\
\hline UPDRS III & $54 \%(0.001)$ & $50 \%(0.00001)$ & $54 \%$ & 0.0001 & $43 \%(0.001)$ \\
\hline Temblor & $81 \%(0.001)$ & $87 \%$ & & 0.0001 & $60 \%(0.03)$ \\
\hline Rigidez & $65 \%(0.001)$ & $59 \%$ & $76 \%$ & 0.0001 & $73 \%(0.01)$ \\
\hline Bradicinesia & $47 \%(0.001)$ & $42 \%$ & & 0.0001 & $25.3 \%(0.04)$ \\
\hline Marcha & $40 \%(0.001)$ & $41 \%$ & $53 \%$ & 0.01 & $35.7 \%(0.002)$ \\
\hline Estabilidad postural & $42 \%(0.001)$ & $31 \%$ & & 0.01 & \\
\hline Disquinesias & $66 \%(1)$ & 0.001 & $79 \%$ & $29 \%(0.02)$ & $47.2 \%(0.02)$ \\
\hline Reducción dopa & $50 \%(2)$ & & & $90 \%(0.0001)$ & $79 \%(0.009)$ \\
\hline
\end{tabular}

(1): duración. (2): incapacidad

como las escalas de actividades de la vida diaria (UPDRS II) en los pacientes intervenidos con NST-DBS en situación "off" medicación mantienen una mejoría significativa a los 4-5 años en los distintos grupos al compararlos con los resultados previos a la cirugía, pero no con los resultados obtenidos al año de la misma ${ }^{37,45,50,53,59}$ (Tabla 2). Los signos clínicos como el temblor (mejoría entre 60-87\%), la rigidez (mejoría entre 59-73\%) y la bradicinesia (mejoría entre $35.7-41 \%$ ) mantuvieron el beneficio estadístico a largo plazo. Las disquinesias inducidas por la medicación, mejoraron entre el 50-90\%, tanto en intensidad de las mismas como en porcentaje del tiempo "on", así como las fluctuaciones motoras que también mantuvieron el beneficio obtenido ${ }^{50,53,59}$. Por último, la medicación permaneció reducida entre un 47.2 y un $59 \%$ en los pacientes intervenidos a los 4-5 años a $^{37,45,50,53,59}$.

Por otro lado, se ha podido apreciar un empeoramiento, o bien una pérdida de la significación estadística, en los signos axiales de la enfermedad como la marcha, la estabilidad postural y el lenguaje en todos los grupos al comparar los resultados a largo plazo con los resultados al año de la cirugía ${ }^{37,45,50,53}$. Esto hace que las escalas de evaluación también declinen con el paso de los años y tanto la UPDRS II y III (escalas de calidad de vida y motora) estén peor a largo plazo al ser comparadas con los resultados al año postoperatorio ${ }^{37,45,50,53}$. Así Krack y cols, han mostrado un empeoramiento entre la UPDRS III del primer año postintervención (mejoría del 65\%) al ser comparados sus resultados con los obtenidos a los 5 años tras la cirugía (mejoría del 54\%) $(\mathrm{p}<0.001)$, al igual que la escala de actividades de la vida diaria UPDRS II (65\% al año y $49 \%$ a los 5 años $)^{37,50}$. Los beneficios observados en los signos axiales aunque persisten, declinan a largo plazo y esto podría estar relacionado con la progresión de la propia enfermedad y con la posible afectación de sistemas no dopaminérgicos que afecten a otros circuitos neuronales en el estadio avanzado en que la EP se encuentra (Tabla 2).

\section{Efecto de la estimulación del NST en el "on" medicación}

La UPDRS motora, en los pacientes intervenidos en situación "on/on" (estimulación y medicación) a largo plazo mostró un deterioro significativo con la situación "on/on" al año de la cirugía y un beneficio clínico aunque no estadístico respecto al "on" preoperatorio ${ }^{37,45,50,53,59}$.

\section{Efectos adversos relacionados con la estimulación}

Además de las complicaciones referidas por los distintos grupos y directamente relacionadas con el sistema implantado, (electrodos, batería y cables de conexión) que pueden ser de $4.5 \%{ }^{26}$, existen otras complicaciones relacionadas bien con la estimulación en la diana quirúrgica implantada, NST, o con la evolución de la propia enfermedad. Así se ha descrito pacientes que tras cirugía del NST-DBS han presentado una desinhibición transitoria ${ }^{30}$, manías $^{28,37}$, 
depresión $^{6,17,37,45,50,53}$, intentos de suicidio ${ }^{30,53}$, alucinaciones y delirio ${ }^{50}$, apatía transitoria y permanente ${ }^{37,53}$, e hipersexualidad $^{59}$ con la estimulación. Estas complicaciones de tipo psiquiátrico, también pueden encontrarse en pacientes no intervenidos y con una enfermedad en estado avanzado y por tanto es difícil conocer si las mismas pueden ser debidas a la estimulación eléctrica en zonas asociativas o límbicas dentro del propio núcleo o bien a la propia enfermedad $^{40}$. Por otro lado, la reducción de la medicación tras la intervención quirúrgica, también podría jugar un papel en alguno de los cuadros psiquiátricos que estos pacientes presentan ${ }^{37}$. Por tanto, actualmente no se conoce bien la evolución de los pacientes con EP que presentan problemas de tipo psiquiátricos tras la cirugía, ya que los mismos podrían ser inherentes a la propia enfermedad más que deberse al efecto adverso de la propia estimulación.

La depresión tras la intervención con NST-DBS, ha sido descrita con una incidencia variable, entre $1.5 \%$ hasta un $25 \%$ de los pacientes operados ${ }^{64}$ y su interpretación no es clara, ya que la EP conlleva una alta incidencia de pacientes con depresión y sin ninguna relación con el procedimiento quirúrgico. Así Houeto y cols observaron que los pacientes que presentaron un síndrome depresivo tras la cirugía, fueron los que presentaron episodios de depresión antes de la intervención ${ }^{30}$. Otros autores al analizar 40 pacientes consecutivos con EP para ser intervenidos con estimulación, describieron que un $60 \%$ de los mismos, presentaban una depresión, $35 \%$ presentaron psicosis $\mathrm{y}$ un $40 \%$ ansiedad. De todos ellos, un $23 \%$ precisaron tratamiento psiquiátrico antes de ser intervenidos ${ }^{63}$. Por otro lado, los cuadros depresivos también pueden estar relacionados con la disminución de la medicación dopaminérgica en los pacientes intervenidos y los mismos suelen ser reversibles al incrementar la misma ${ }^{37}$. Por último, en un estudio realizado por Funkiewicz y cols, los pacientes que previamente a la cirugía presentaron una depresión moderada (14\%), a los 3 años de la intervención empeoraron su cuadro, mientras que un $86 \%$ mejoraron en las escalas de evaluación. Sin embargo, los pacientes que presentaban una depresión severa sólo mejoraron un 50\% tras la cirugía a los 3 años ${ }^{19}$.

Los cambios cognitivos o de humor, aparecen más frecuentemente en pacientes intervenidos en el NST con estimulación que en el grupo de pacientes intervenidos en el GPi, pudiendo tener relación con la distribución de la estimulación hacia las porciones asociativas y límbicas del propio $\mathrm{NST}^{3,50}$. No obstante, son pocos los casos publicados con seguimiento a largo plazo con GPi-DBS ${ }^{50}$ y este dato podría inducir un sesgo a la hora de evaluar las complicaciones de tipo psiquiátrico entre ambas dianas quirúrgicas. Para otros autores, la estimulación del NST no ha mostrado un efecto neuropsicológico sobre el humor en 77 pacientes con EP intervenidos y seguidos durante 3 años ${ }^{19}$.
También se han descrito variaciones de humor en pacientes intervenidos en relación con la disminución de medicación dopaminérgica ${ }^{37}$.

\section{Deterioro cognitivo}

Un problema que se ha podido observar en los pacientes intervenidos con NST-DBS es que un porcentaje de los mismos se deteriora cognitivamente en las evaluaciones a largo plazo. Así Ostergaard y Sunde reportan una incidencia de demencia en el grupo de pacientes intervenidos de un $21 \%$ a los 5 años, bien por pérdida de memoria o por alteraciones de funciones ejecutivas ${ }^{45}$. La edad media del grupo de pacientes intervenido que cursó con demencia fue de 64 años, con 22 años de enfermedad, comparativamente con una media de 59 años de edad y 15 de evolución de los intervenidos que no presentaron esta complicación ${ }^{45}$. En el estudio multicéntrico de los 49 pacientes con NST-DBS, los autores también describieron una pérdida de memoria y alteraciones cognitivas (alucinaciones, depresión, delirio, etc) que ocurrieron en una incidencia del 30\% de los pacientes intervenidos en esta diana ${ }^{50}$. En el estudio, los problemas cognitivos fueron más frecuentes en el grupo intervenido en el NST comparativamente con el grupo intervenido en el GPi ${ }^{50}$.

Krack y cols en los pacientes con estimulación del NST y evaluados a los 5 años, describieron un deterioro en la escala de demencia de Mattis que refleja la aparición del deterioro en tres pacientes registrado entre el tercer y quinto año tras la cirugía, así como una disminución de las funciones del lóbulo frontal ${ }^{37}$. Otras evaluaciones realizadas por otros grupos, también han mostrado un deterioro significativo cognitivo a los 3 y 5 años del implante aunque la incidencia no parece ser mayor que en pacientes tratados médicamente y no intervenidos durante el mismo periodo de tiempo ${ }^{4,53}$. Se ha descrito que la incidencia de demencia en los pacientes con EP puede alcanzar el 60\% a los 10-15 años desde la aparición de los problemas motores y que la misma podría ser 6 veces superior a pacientes control ${ }^{1,52}$.

Otros autores, por el contrario, no han encontrado deterioro cognitivo en las escalas de evaluación en los pacientes intervenidos con NST-DBS. Así Contarino y cols han publicado que 6 de 11 pacientes intervenidos a los 5 años presentaron problemas en la fluencia verbal pero no observaron ningún deterioro cognitivo en la batería de test realizados ${ }^{15}$. Funkiewiez y cols en un grupo de pacientes a los 3 años de la cirugía tampoco mostraron ningún deterioro en los diferentes test estudiados ${ }^{19}$. Por último, SaintCyr y cols tras una larga batería de test neuropsicológicos, concluyeron en un estudio con 11 pacientes, que la cirugía del NST podía tener un impacto negativo en los test frontales ejecutivos sobre todo en los pacientes mayores de 69 años $^{51}$. Por tanto los estudios publicados no llegan a resol- 
ver las controversias existentes sobre el posible deterioro cognitivo en los tests con implicación de la función ejecutiva y si este deterioro puede ser secundario a la cirugía subtalámica o bien deberse a otros factores de la evolución de la propia enfermedad. La conclusión podría ser que la estimulación del NST puede inducir cambios cognitivos y de humor de poca repercusión global si los comparamos con el gran beneficio motor que se obtiene ${ }^{55}$. Por otro lado, tampoco podemos olvidar que los deterioros cognitivos también aparecen en los pacientes no sometidos a cirugía que conllevan una enfermedad crónica como la EP durante años.

\section{¿Es la cirugía de estimulación del NST neuroprotectora?}

Cuando se analiza críticamente la estimulación del NST en pacientes con EP de larga evolución, han de ser considerados varios factores. Según los resultados de las series, la estimulación del NST es eficaz para el control sintomático de la EP (evidencia tipo I) aunque su efecto sobre las fluctuaciones motoras y las disquinesias no muestra datos concluyentes debido al factor introducido por la disminución de la medicación que ocurre en la mayoría de los pacientes tras la intervención ${ }^{22}$. Los pacientes de las series quirúrgicas, presentan complicaciones motoras previas a la cirugía debido a la larga evolución de la enfermedad (15 años de media) así como a la múltiple farmacología empleada. La cirugía ha mostrado ser superior al óptimo tratamiento médico posible, como se ha reportado en dos estudios aleatorizados enrolando a más de 400 pacientes y con un seguimiento de 6 meses $^{16,66}$. La UPDRS motora del grupo quirúrgico mejoró significativamente $(\mathrm{p}<0.001)$ en comparación con el no intervenido, aunque los efectos adversos serios fueron superiores en el grupo quirúrgico (13\% vs 4\%). En el estudio europeo, 3 pacientes del grupo quirúrgico fallecieron, uno de ellos por un hematoma intraparenquimatoso durante la cirugía. Otro de los pacientes tuvo una neumonía 6 semanas después de entrar en la randomización y un tercero se suicidó. En el grupo de pacientes no intervenido también hubo un fallecimiento de un paciente durante un estado psicótico ${ }^{16}$. En el grupo americano 2 pacientes del grupo quirúrgico fallecieron uno por una hemorragia 24 horas después de la intervención y un segundo por un tumor pulmonar ${ }^{66}$. La frecuencia de efectos adversos no serios fue mayor en el grupo no quirúrgico $(64 \% \text { frente } 50 \% \mathrm{p}<0.08)^{16}$.

La estimulación del NST no parece ser neuroprotectora, según se ha publicado en un estudio prospectivo realizado en 30 pacientes intervenidos con cirugía bilateral y con buena respuesta clínica $^{32}$. En los pacientes tratados quirúrgicamente los estudios con PET (tomografía por emisión de positrones) han mostrado la misma progresión de la enfermedad al ser comparada con otro grupo de pacientes no intervenidos y seguidos en un estudio longitudinal. La evaluación ha sido llevada durante 12-36 meses ${ }^{32}$. Por tanto, el que la cirugía del NST no tenga un efecto neuroprotector es un resultado que debemos de considerarlo dentro de lo previsto, ya que los pacientes en el estadio que se intervienen tienen una importante depleción DA tras los años de enfermedad, tanto por la pérdida neuronal dopaminérgica de la SNc, como una gran denervación estriatal derivada de la pérdida de sus terminales de proyección. El estriado denervado con respuesta patológica a la concentración plasmática de levodopa fluctúa en relación a su dosificación, conllevando los fenómenos conocidos como respuesta "on-off", fluctuaciones motoras y disquinesias ${ }^{43}$. A pesar de este resultado en contra del efecto neuroprotector de la estimulación subtalámica, en los diferentes estudios a largo plazo previamente descritos, la situación motora en el "off/off" postquirúrgico de los pacientes no es peor que en el "off" preoperatorio a pesar del periodo transcurrido desde la intervención quirúrgica ${ }^{37,45,50,53}$. Podemos concluir que ninguna cirugía, por buenos resultados clínicos que obtenga, puede ser considerada como neuroprotectora al realizarse tardíamente. Sería necesario mantener una inervación estriatal y por tanto una falta de complicaciones farmacológicas para poder evaluar el resultado de una cirugía realizada más precozmente. Por otro lado, se ha podido comprobar que el beneficio obtenido tras la cirugía, no está relacionado con la liberación endógena de DA estriatal por la falta de desplazamiento del (C) raclopride en PET realizados a pacientes implantados ${ }^{2}$.

\section{EI PPN como nueva diana quirúrgica}

El núcleo pendúnculo pontino (PPN) está situado en el tronco cerebral, en lo que se conoce como región motora mesencefálica, pudiendo tener un papel importante en la iniciación y control de la marcha en animales de experimentación ${ }^{20,46}$. Se ha podido comprobar que el PPN en la EP sufre una degeneración neuronal y paralelamente, los macacos tratados con MPTP a los que se les lesionó el PPN, empeoraron la acinesia ${ }^{46}$ y por el contrario, en primates con parkinsonismo inducido, la estimulación del PPN indujo una mejoría en la misma ${ }^{33}$. Basados en estos datos 6 pacientes con EP y con mal control farmacológico, con signos preferentemente axiales han sido intervenidos con estimulación del PPN y del NST con un seguimiento de 6 meses $^{56 .}$ La mejoría del PPN-DBS con estimulación a baja frecuencia $(25 \mathrm{~Hz})$ a los 3-6 meses ha sido de un 32\% en la UPDRS motora $(\mathrm{p}<0.05)$, mientras que la mejoría del STN-DBS (alta frecuencia) ha sido de un $54 \%(\mathrm{p}<0.01)$. La suma de las dos estimulaciones también ha mostrado un importante beneficio en las escalas de evaluación $(p<0.01)$. La mejoría experimentada con la estimulación del PPN ha sido significativa para los signos axiales en los 6 pacientes 
descritos $(\mathrm{p}<0.01)^{56}$. El impacto de la estimulación del PPN es menor al de la estimulación del NST y actualmente no se plantea esta diana quirúrgica como alternativa al NST. Sin embargo, en pacientes que consideramos como no susceptibles de ser intervenidos en el NST, como son los pacientes con problemas de la marcha y estabilidad postural en el "on" farmacológico podrían ser candidatos a esta nueva diana. Varios grupos quirúrgicos han iniciado un protocolo para tratar pacientes con EP con un predominio axial, así como pacientes con parálisis supranuclear progresiva (PSP) con PPN-DBS como única diana quirúrgica, pero en la actualidad los estudios están en su fase inicial y no se dispone de datos clínicos analizados sobre este tratamiento.

\section{Cirugía precoz de estimulación del NST en la EP}

Actualmente con los nuevos fármacos disponibles y la mejora en la calidad de vida de los pacientes, se puede conocer la historia natural de la EP a largo plazo. Antes de introducir nuevas dianas quirúrgicas para los signos clínicos con una menor respuesta a la cirugía, como son los signos axiales y previamente a justificar problemas psiquiátricos como inherentes a la estimulación, debemos de pensar en una cirugía más precoz en los pacientes con EP. La media en años desde el diagnóstico de la enfermedad al momento de la cirugía en los diferentes grupos ha sido de 15 años $^{37,45,50,53,59}$. El tratamiento quirúrgico ofrece una alternativa a los pacientes cuando comienzan a presentar fluctuaciones motoras a la levodopa y precisan incrementar su dosificación y añadir agonistas dopaminérgicos y como hemos comentado, la estimulación es significativamente superior al óptimo tratamiento médico ${ }^{16,66}$. Por otro lado, es conocido que un importante porcentaje de pacientes con EP tendrá un deterioro cognitivo que les llevará a una demencia con la evolución de la enfermedad.

La cirugía cuando los pacientes presentan complicaciones relacionadas con el estado avanzado de su proceso induce un beneficio que puede ser corto en años, ya que desde la intervención hasta el deterioro cognitivo puede pasar un tiempo de 5-7 años. La cirugía precoz (5-7 años desde el diagnóstico) podría ser una oferta que prolongaría los años de beneficio motor y de la calidad de vida de los pacientes durante un periodo más largo. Las complicaciones quirúrgicas no estarían incrementadas por adelantar la misma, sino lo opuesto, ya que se intervendrían pacientes más jóvenes y con menor incidencia de complicaciones psiquiátricas. Por otro lado, tras la cirugía precoz, complicaciones como fluctuaciones motoras y disquinesias tampoco ocurrirían en el mismo porcentaje, ya que la estimulación del NST generalmente conlleva una reducción en la cantidad de medicación.

Con este criterio se ha publicado un estudio comparativo en 20 pacientes con EP de corta duración (6.8 años desde el inicio y UPDRS motora de 29 de media). El estudio ha sido prospectivo y aleatorizado entre NST-DBS (10 pacientes) contra el óptimo tratamiento médico (10 pacientes) con un seguimiento entre 6 y 18 meses $^{54}$. La UPDRS II (escala de calidad de vida) mejoró un $24 \%$ en el grupo quirúrgico y un $0 \%$ en el tratado médicamente $(\mathrm{p}<0.05)$. A los 18 meses la UPDRS motora así como las complicaciones de la medicación y la dosis de la misma se redujeron en un $69 \%, 83 \%$ y $57 \%$ respectivamente en los pacientes intervenidos, mientras empeoraron un $29 \%, 15 \%$ y $12 \%$ en el grupo tratado farmacológicamente. El resultado final del estudio ha mostrado que la cirugía, NST-DBS, en pacientes con pocos años de enfermedad es significativamente superior al òptimo tratamiento médico ${ }^{54}$. Según este estudio francés, los pacientes podrían beneficiarse durante un mayor tiempo del beneficio motor, ya que tendrán un mayor número de años con buena calidad de vida antes de que aparezcan nuevas complicaciones inherentes a la progresión de la enfermedad. Actualmente se está realizando un estudio similar al expuesto con un mayor número de pacientes cuyos resultados están todavía pendientes de ser evaluados.

\section{Conclusiones}

La estimulación del NST en pacientes con EP y con complicaciones motoras mejora los signos cardinales de la enfermedad significativamente al ser comparada con el óptimo tratamiento médico y el beneficio se mantiene a largo plazo. Sin embargo, el beneficio de la estimulación decae con el paso de los años sobre todo en los signos axiales y el lenguaje, posiblemente relacionado con la evolución de la propia enfermedad. En los pacientes con una EP compleja y de larga evolución las complicaciones motoras, como las fluctuaciones y disquinesias, así como los problemas psiquiátricos y un deterioro cognitivo ocurren. La cirugía realizada precozmente desde el diagnóstico, puede mejorar estas complicaciones así como la calidad de vida durante un tiempo más largo sin un incremento de los riesgos quirúrgicos.

\section{Bibliografía}

1. Aasland, D., Andersen, K., Larsen, J.P., Lolk, A., Nielsen, H., Kragh-Sorensen, P.: Risk of dementia in Parkinson's disease: a community-based, prospective study. Neurology 2001; 56: 730-736.

2. Abosch, A., Kapur, S., Lang, A,E., Hussey, D., Sime, E., Miyasaki, J., Houle, S., Lozano, AM.: Stimulation of the subthalamic nucleus in Parkinson's disease does not produce striatal dopamine release. Neurosurgery 2003; 53: 1095-1105.

3. Anderson, V.C., Burchiel, K.J., Hogarth, P., Favre, J., Hammerstad, J.P.: Pallidal vs subthalamic nucleus deep brain 
stimulation in Parkinson disease. Arch Neurol 2005; 62: 554560.

4. Aybeck, S., Gronchi-Perrin, A., Berney, A., Chiuvé, S.C., Villemure, J-G., Burkhard, P.R., Vingerhoets, J.G.: Long-term cognitive profile and incidence of dementia after STN-DBS in Parkinson's disease. Mov Disord 2007; 22: $974-$ 981.

5. Aziz, T.Z., Peggs, D., Sambrook, M.A., Crossman, A.R.: Lesion of the subthalamic nucleus for the alleviation of MPTP-induced parkinsonism in the primate. Mov Disord 1991; 6: 288-293.

6. Bejjani, B.P., Damier, P., Arnulf, I., Thivard, L.: Transient acute depression induced by high-frequency deep-brain stimulation. N Engl J Med 1999; 340: 1476-1480.

7. Bejjani, B.P., Gervais, D., Arnulf, I., Papadopoulos, S., Demeret, S., Bonnet, A.N., Cornu, P., Agid, Y.: Axial parkinsonian symptoms can be improved: the role of levodopa and bilateral subthalamic stimulation. J Neurol Neurosurg Psychiatry 2000; 68: 595-600.

8. Benabid, A.L., Pollak, P., Hommel, M., Gao, J.M., de Rougemont, J., Perret, T.: Traitement du tremblement parkinsonien par stimulation chronique du noyau ventral intermediére du thalamus. Rev Neurol (Paris) 1989; 145: 320-323.

9. Benabid, A.L., Pollak, P., Seigneuret, E., Hoffmann, D., Gay, E., Perret, J.: Chronic VIM thalamic stimulation in Parkinson's disease, essential tremor and extrapiramidal dyskinesias. Acta Neurochir (Wien) Suppl 1993; 58: 39-44.

10. Benabid, A.L., Pollak, P., Gross, C., Benazzouz, A., Gao, D.M., Laurent, A., Gentil, M., Perret, J.: Accute and long term effects of subthalamic nucleus stimulation in Parkinson's disease. Stereotac Funct Neurosurg 1994; 62: 76-84.

11. Benazzouz, A., Gross, C., Feger, J., Boraud, T,. Bioulac, B.: Reversal of rigidity and improvement in motor pèrformance by subthalamic high-frequency stimulation in MPTP-treated monkeys. Eur J Neurosci 1993; 5: 382-389.

12. Bergman, H., Wichmann, T., DeLong, M.R.: Reversal of experimental parkinsonism by lesions of the subthalamic nucleus. Science 1990; 249: 1436-1438.

13. Bergman, H., Wichmann, T., Karmon, B., DeLong, M.R.: The primate subthalamic nucleus.II. Neuronal activity in the MPTP model of parkinsonism. J Neurophysiol 1994; 72: 507-519.

14. Charles, P.D., Van Blercom, N., Krack, P., Lee, S.L., Xie, J., Besson, G., Benabid, A.L., Pollak, P.: Predictors of effective bilateral subthalamic nucleus stimulation for PD. Neurology 2002; 59: 932-934.

15. Contarino, M.F., Daniele, A., Sibilia, A.H., Romito, L.M., Betivoglio, A.R., Gainotti, G., Albanese, A.: Cognitive outcome 5 years after bilateral chronic stimulation of subthalamic nucleus in patients with Parkinson's disease. J Neurol Neurosurg Psychiatry 2007; 78: 248-252.

16. Deuschl, G., Schade-Brttinger, C., Krack, P., Volkmann, J., Schafer, H., Bötzel, K., Daniels, C., y cols.: A rando- mized trail of deep-brain stimulation for Parkinson's disease. N Engl J Med 2006; 355: 896-908.

17. Doshi, P.K., Chaya, N., Bhatt, M.H.: Depression leading to attempted suicide after bilateral subthalamic nucleus stimulation for Parkinson's disease. Mov Disord 2002; 17 : 1084-1085.

18. Figueiras-Méndez, R., Marín-Zarza, F., Molina, A., Jiménez-Jiménez, F.J., Orti-Parga, M., Magariños-Ascone, C., López-Pina, M.A., Martínez, V.: Subthalamic nucleus stimulation improves directly levodopa induced dyskinesias in Parkinson's disease. J Neurol Neurosurg Psychiatry 1999; 66: 549-550.

19. Funkiewiez, A., Andouin, C., Caputo, E., Krack, P., Fraix, V., Klinger, H.: Long term effects of bilateral subthalamic nucleus stimulation on cognitive function, mood, and behaviour in Parkinson's disease. J Neurol Neurosurg Psychiatry 2004; 75: 834-839.

20. García-Rill, E.: The pedunculopontine nucleus (Review). Prog Neurobiol 1991; 36: 363-389.

21. Ghika, J., Villemure, J-G., Frankhauser, H., Favre, J., Assal, G.: Efficiency and safety of bilateral contempouraneous pallidal stimulation (deep brain stimulation) in levodopa-responsive patients with Parkinson's disease with severe motor fluctuations: a 2-year follow-up review. J Neurosurg 1998; 89: 713-718.

22. Goetz, C.G., Poewe, W., Rascol, O., Sampaio, C.: Evidence-based medical review update: Pharmacological and surgical treatment of Parkinson's disease: 2001 to 2004. Mov Disord 2005; 20: 523-539.

23. Guridi, J., Herrero, M.T., Luquin, M.R., Guillén, J., Ruberg, M., Laguna, J., et al.: Subthalamotomy in parkinsonian monkeys. Behavioural and biochemical analysis. Brain 1996; 119: 1717-1727.

24. Guridi, J., Rodríguez-Oroz, M.C., Manrique, M.: Tratamiento quirúrgico de la enfermedad de Parkinson. Neurocirugía 2004; 15: 5-16.

25. Guridi, J., Obeso, J.A., Rodríguez-Oroz, M.C., Lozano, A.M., Manrique, M.: Levodopa induced dyskinesias and stereotactic surgery for Parkinson's disease. Neurosurgery 2008; 62: 311-325.

26. Hamani, C., Richter, E., Schwalb, J., Lozano, A.M.: Bilateral subthalamic nucleus stimulation for Parkinson's disease: a systematic review of the clinical literature. Neurosurgery 2005; 56: 1313-1324.

27. Hariz, M.I., Rehncrona, S., Quinn, N., Speelman, J.D.: Wensing $\mathrm{C}$ and the Multicenter Advanced Parkinson's disease Deep Brain Stimulation Group. Adverse events at 3-4 years follow-up in patients with parkinson's disease treated with deep brain stimulation in the globus pallidus interna or in the subthalamic nucleus. An independent assessment. Mov Disord 2008; 23: 416-421.

28. Herzog, J., Reiff, J., Krack, P., Witt, K.: Manic episode with psicotic symptoms induced by subthalamic nucleus 
stimulation in patients with Parkinson's disease. Mov Disord 2003; 18: 1382-1384.

29. Houeto, J.L., Bejjani, P.B., Damier, P.: Failure of longterm pallidal stimulation corrected by subthalamic stimulation in PD. Neurology 2000; 55: 728-730.

30. Houeto, J.L., Mallet, L., Mesnage, V., Tezenas du Montcel, S., Behar, C., Gargiulo, M., Torny, F., et al.: Subthalamic stimulation in Parkinson disease: behavior and social adaptation. Arch Neurol 2006; 63: 1090-1095.

31. Houeto, J.L., Damier, P., Bejjani, P.B., Staedler, C., Bonnet, M., Arnulf, I., Pidoux, B., Dormont, D., Cornu, P., Agid, Y.: Subthalamic stimulation in Parkinson's disease. A multidisciplinary Approach. Arch Neurol 2000; 57: 461-465.

32. Hilker, R., Portman, A.T., Voges, J., Staal, M.J., Burghaus, L., van Laar, T., Koulousakis, A., Maguire, R.P., Pruim, J., de Jong, B.M., Herholz, K., Sturm, V., Heiss, WD., Leenders, K.L.: Disease progression continues in patients with advanced Parkinson's disease and effective subthalamic nucleus. J Neurol, Neurosurg Psychiatry 2005; 76: 12171221.

33. Jenkinson, N., Nandi, D., Miall, R.C., Stein, J.F., Aziz, T.Z.: Pedunculopontine nucleus stimulation improves akinesia in a parkinsonian monkey. Neuroreport 2004; 15: 2621-2624.

34. Katayama, Y., Kasai, M., Oshima, H., Fukaya, C., Yamamoto, T., Mizutani, T.: Double blinded evaluation of the effects of pallidal and subthalamic nucleus stimulation on daytime activity in advanced Parkinson's disease. Parkinsonism Relat Disord 2001; 7: 35-40.

35. Krack, P., Pollak, P., Limousin, P., Hoffmann, D., Xie, J., et al.: Subthalamic nucleus or internal pallidal stimulation in young onset Parkinson's disease. Brain 1998; 121: 451457.

36. Krack,, P., Pollak, P., Limousin, P., Bennazouz, A., Deuschl, G., Benabid, A.L.: From off-period dystonia to peakdose chorea: the clinical spectrum of variying subthalamic nucleus activity. Brain 1999; 122: 1133-1146.

37. Krack, P., Batir, A., Van Blercom, N., Chabardes, S., Fraix, V., Ardouin, C., et al.: Five-year follow-up of bilateral stimulation of the subthalamic nucleus in advanced Parkinson's disease. N Engl J Med 2003; 349: 1925-1934.

38. Krause, M., Fogel, W., Heck, A., Hacke, W., Bonsanto, M., et al.: Deep brain stimulation for the treatment of Parkinson's disease: subthalamic nucleus versus globus pallidus internus. J Neurol Neurosurg Psychiatry 2001; 70 : 464-470.

39. Kumar, R., Lang, A.E., Rodríguez-Oroz, M.C., Lozano, A.M., Limousin, P., Pollak, P., Benabid, A.L., Guridi, J., Ramos, E., et al.: Deep brain stimulation of the globus pallidus pars interna in advanced Parkinson's disease. Neurology 2000; 55 (Suppl 6): 34-39.

40. Lang, A.E., Obeso, J.A.: Challenges in Parkinson's disease: restoration of the nigrostriatal dopamine system is not enough. Lancet Neurol 2004;5: 309-316.
41. Molinuevo, J., Valdeoriola, F., Tolosa, E., Rumiá, J., Vals-Sole, J., Roldán, H., Ferrer, E.: Levodopa withdrawal after bilateral subthalamic nucleus stimulation in advanced Parkinson disease. Arch Neurol 2000; 57: 983-988.

42. Obeso, J.A., Guridi, J., Rodríguez-Oroz, M.C., Agid, Y., Bejjani, P., Bonnet, A.M., Lang, A.M., Lozano, A.M., Kumar, R., et al.: Deep-Brain stimulation of the subthalamic nucleus or the pars interna of tha globus pallidus in Parkinson's disease. N Engl J Med 2001; 345: 956-963.

43. Obeso, J.A., Rodríguez-Oroz, M.C., Marín, C., Alonso, F., Zamarbide, I., Lanciego, J.L., Rodríguez-Díaz, M.: The origin of motor fluctuations in Parkinson's disease: importance of dopaminergic innervation and basal ganglia circiuts. Neurology 2004; 62 (Suppl 1): 17-30.

44. Okun, M.S., Foote, K.D.: Subthalamic nucleus vs globus pallidus interna deep brain stimulation, the rematch. (Editorial). Arch Neurol 2005; 62: 533-536.

45. Ostergaard, K., Aa Sunde, N.: Evolution of Parkinson's disease during 4 years of bilateral deep brain stimulation of the subthalamic nucleus. Mov Disord 2006; 21: 624-631.

46. Pahapill, P.A., Lozano, AM.: The pedunculopontine nucleus and Parkinson's disease. Brain 2000; 123: 17671783.

47. Pollak, P., Benabid, A.L., Gross, C., Gao, D.M., Laurent, A., Benazzouz, A., Hoffmann, D., et al.: Effects of the stimulation of the subthalamic nucleus in Parkinson disease. Rev Neurol (Paris) 1993;149:175-176.

48. Rodríguez-Oroz, M.C., Gorospe, A., Guridi, J., Ramos, E., Linazasoro, G., Rodríguez Palmero, M., Obeso, J.A.: Bilateral deep brain stimulation of the subthalamic nucleus in Parkinson's disease. Neurology 2000; 55 (Suppl): 45-51.

49. Rodríguez-Oroz, M.C., Zamarbide, I., Guridi, J., Palmero, M., Obeso, J.A.: Efficacy of deep brain stimulation of the subthalamic nucleus in Parkinson's disease 4 years after surgery: double blind and open evaluation. J Neurol Neurosurg Psychiatry 2004; 75: 1382-1385.

50. Rodríguez-Oroz, M.C., Obeso, J.A., Lang, A.E., Houeto, J.L., Pollak, P., Rehncrona, S., Kulisevsky, J., et al.: Bilateral deep brain stimulation in Parkinson's disease: a multicentre study with 4 years follow-up. Brain 2005; 128: 22402249.

51. Saint-Cyr, J.A., Trepanier, L., Kumar, R., Lozano, A.M.: Neuropsycological consequences of chronic bilateral stimulation of the subthalamic nucleus in Parkinson's disease. Brain 2000; 123: 2091-2108.

52. Schmidt, R., Freidl, W., Fazekas, F., Reinhart, B., Grieshofer, P., Koch, M., Eber, B., Schumacher, M., Polmin, K., Lechner, H.: The Mattis dementia Rating Scale: normative data from 1001 healty volontary. Neurology 1994; 44: 964966.

53. Schüpbach,, W.M.M., Chastan, N., Welter, M.L., Houeto, J.L., Masnage, V., Bonnet, A.M., Czernecki, V., Maltete, D., Hartmann, A., Mallet, L., Pidoux, B., Dormont, D., 
Navarro, S., Cornu, P., Mallet, A., Agid, Y.: Stimulation of the subthalamic nucleus in Parkinson's disease: a 5 year follow up. J Neurol Neurosurg Psychiatry 2005; 76: 1640-1644.

54. Schüpbach, W.M.M., Matête, D., Houeto, J.L., Tezenas du Montcel, S., Mallet, L., Welter, M.L., Gargiulo, M., y cols.: Neurosurgery at an early stage of Parkinson disease. A randomized, controlled trial. Neurology 2007; 68: 267-271.

55. Speelman, J.D., Smeding, H.M.M., Schmand, B.: Does chronic subthalamic nucleus stimulation in advanced Parkinson's disease cause invalidating cognitive and behavioural dysfunctions? J Neurol Neurosurg Psychiatry 2007; 78: 221 (Editorial).

56. Stefani, A., Lozano, A.M., Peppe, A., Stanzione, P., Gelati, S., Tropepi, D., Pierantozzi, L.B., Scarnati, E., Mazzone, P.: Bilateral deep brain stimulation of the pedunculopontine and subthalamic nuclei in severe Parkinson's disease. Brain 2007; 130: 1596-1607.

57. Vila, M., Levy, R., Herrero, M.T., Ruberg, M., Facheux, B., Obeso, JA.: Consequences of nigrostriatal denervation on the functioning of the basal ganglia in human and nonhuman primates: an in situ hybridization study of cytocrome oxidase subunit 1 mRNA. J Neurosci 1997; 17: 765-773.

58. Vingerhoets, F.J.G., Villemure, J.G., Temperli, P., Pollo, C., Pralong, E., Ghika, J.: Subthalamic DBS replaces levodopa in Parkinson's disease. Two year follow-up. Neurology 2002; 58: 396-401.

59. Visser-Vanderwalle, V., van der Linden, C., Temel, Y. Celik, H., Ackermans, L., Spincemaille, G., Caemaert, J.: Long-term of bilateral subthalamic nucleus stimulation in advanced Parkinson's disease: a four year follow-up study. Parkinsonism Relat Disord 2005; 11: 157-165.

60. Vitek, J.L.: Deep brain stimulation for Parkinson's disease. Sterotact Funct Neurosurg 2002; 78: 119-131.
61. Volkmann, J., Allert, N., Voges, J., Weiss, P.H., Freund, H-J., Sturm, V.: Safety and efficacy of pallidal or subthalamic nucleus stimulation in advanced PD. Neurology 2001; 56: 548-551.

62. Volkmann, J., Allert, N., Voges, J., Sturm, V., Schnitzler, A., et al.: Long-term results of bilateral pallidal stimulation in Parkinson's disease. Ann Neurol 2004; 55: 871-875.

63. Voon, V., Saint-Cyr, J., Lozano, A.M., Moro, E., Poon, Y.Y., Lang, A.E.: Psychiatric symptoms in patients with Parkinson disease presenting for deep brain stimulation surgery. J Neurosurg 2005; 103: 246-251.

64. Voon, V., Kubu, C., Krack, P., Houeto, J-L., Tröster, A.: Deep brain stimulation: neuropsychological and neuropsychiatric issues. Mov Disord 2006; 21 (Supl 14) 305-327.

65. Weaver, F., Follet, K., Hur, K., Ippolito, D., Stern, M.: Deep brain stimulation in Parkinson's disease: a metaanalysis of patients outcomes. J Neurosurg 2005; 103: 956-967.

66. Weaver, F.M., Follet, K., Stern, M., Hur, K., Harris, C., Marks, W.J., Rothlind, J., Sagher, O., Reda, D., Moy, C., Pahwa, R., Burchiel, K., Hogart, P., Lai, E.C., Duda, J., Holloway, K., Samii, A., Horn, S., Bronstein, J., Stoner, G., Heemskerk, J., Huang, G.: Bilateral deep brain stimulation vs best medical therapy for patients with advanced Parkinson disease. JAMA 2008; 301: 63-73.

Guridi, J.; Rodríguez-Oroz, M.C.; Clavero, P.: Manrique, M.: Revisión crítica de la estimulación subtalámica en la enfermedad de Parkinson. Neurocirugía 2009; 20: 521532 .

Correspondencia: Dr Jorge Guridi. Departamento de Neurocirugía. Clinica Universitaria. Pio XII sn. Pamplona 31008.

Jguridi@unav.es 